\title{
Amartya Sen, teoría de un desarrollo integral, aportes para la superación de la pobreza extrema y construcción de paz en los Montes de María.
}

\author{
Amartya Sen, theory of an integral development, contributions for the \\ overcoming of the extreme poverty and construction of peace in the Mounts \\ of María.
}

\author{
Autores: Georgina Isabel De León Vargas ${ }^{1}$ \\ Damaris Margarita Ballestas León ${ }^{2}$ Fredy Martinez Martinez ${ }^{3}$ \\ Correspondencia: georgina.deleon@curnvirtual.edu.co
}

\begin{abstract}
RESUMEN
En el presente artículo de investigación surge del resultado de un primer acercamiento a la temática del desarrollo integral desde Amatya Sen como estrategia para la superación de la pobreza extrema en Colombia y en consecuencia la construcción de una paz estable y duradera, fundada en oportunidades y equidad para los y las colombianas. Es importante destacar que la pobreza no solo implica bajos niveles de ingreso, usualmente también está acompañada por altas tasas de mortalidad infantil, vulneración del acceso de los derechos fundamentales y reducidas capacidades y libertades para conectarse con todo el territorio. En consecuencia, reducir la pobreza y lograr una mayor equidad requiere mejorar la conexión de las poblaciones con los circuitos del crecimiento económico, y su acceso a bienes y servicios que mejoran sus condiciones de vida pero a su vez incentivar la generación de oportunidades para la consecución de un desarrollo económico y social.
\end{abstract}

Comprender la importancia de la planificación en todos los niveles (nacional, departamental y municipal) y a su vez la concordancia de estos, es preciso si lo que se busca es analizar como las categorías de desarrollo y pobreza se articulan y permiten la posibilidad de ser una oportunidad o un limitante para la construcción de paz. El principal objetivo es conocer cómo se construye la paz en los municipios de San Juan Nepomuceno y el Carmen de Bolívar y hasta qué punto la pobreza puede ser un limitante de esta.

Palabras Claves:

Pobreza extrema, desarrollo, paz, equidad

\footnotetext{
${ }^{1}$ Abogada, Magíster en Derecho laboral Universidad Autónoma de Guerrero México, Convalidada Por el Ministerio de Educación Nacional Resolución 18920 del 18 de Noviembre 2015, Docente tiempo completo, con Función de Coordinación de Investigación del Programa de Derecho de la Corporación Universitaria Rafael Núñez sede Cartagena.

${ }^{2}$ Trabajadora Social, Estudiante Semillerista estudiante V semestre del Programa de Derecho de la Corporación Universitaria Rafael Núñez.

${ }^{3}$ Estudiante semillerista del Programa de Derecho de la Corporación Universitaria Rafael Núñez.
} 
Vol.9, No 1. Diciembre de 2017 pp. 33-52

\begin{abstract}
The research that is proposed arises from the result of a first approach to the theme of integral development of Amatya Sen as a strategy for overcoming extreme poverty in Colombia and consequently the construction of a stable and lasting peace, founded in opportunities and equity for Colombians. It is important to emphasize that poverty not only implies low levels of income, it is also accompanied by high rates of infant mortality, vulnerability of access to fundamental rights and reduces capacities and freedoms to connect the whole territory. Consequently, reducing poverty and achieving greater equity requires the connection of populations with the circuits of economic growth, and their access to goods and services that improve their living conditions but in turn encourage the generation of opportunities for attainment of economic and social development.

Understanding the importance of planning at all levels (national, municipal and municipal) and, in turn, the agreement of these, it is necessary that what is sought is to analyze how the categories of development and poverty are articulated and allow the possibility of being An Opportunity or a Limit for peacebuilding.. The main objective is to know how peace is built in the municipalities of San Juan Nepomuceno and the Carmen de Bolivar and to what extent poverty can be a limit of this.
\end{abstract}

\title{
Keywords:
}

Extreme poverty, Development, peace, equity.

\section{Introducción.}

¿Cuál es el vínculo de los programas Colombia en paz y Colombia equitativa y sin pobreza extrema del Plan Nacional de Desarrollo 2014-2018 y el avance en la construcción de paz territorial a la luz de la teoría de desarrollo de Amartya Sen?

La problemática de la pobreza extrema en Colombia, así como la construcción de paz territorial en el país, debe ser abordada teniendo en cuenta que el desarrollo económico debe permitir un desarrollo integral de la ciudadanía. Como aspecto relevante que da a lugar en el desarrollo de esta investigación se connota una comparación de las situaciones de pobreza-desigualdad y estados en guerra. Realizando un análisis comparativo de las tres categorías. Es objetivo de esta investigación comprobar que el desarrollo económico debe ir de la mano del cumplimiento de las libertades fundamentales de los individuos para la consecución de un desarrollo integral. 
Analizar la concordancia entre los programas Colombia en paz y Colombia equitativa y sin pobreza extrema del plan de desarrollo propuesto por el gobierno del presidente Juan Manuel Santos (2014-2018) a la luz de la teoría de Amartya Sen, analizando si la anterior teoría es de aporte fundamental e identificando como se visiona y materializa el desarrollo en el territorio. La metodología propuesta para ella será de carácter cualitativo, porque las técnicas e instrumentos se retomarán desde este enfoque. Gradualmente se definirá como explicativa. Jurídicamente se ubica en investigación aplicada, puesto que incursionará entre la relación del derecho y el Estado colombiano, a partir del sentido político que tiene la juricidad, con el fin de generar propuestas encaminadas a la transformación social y a la superación del estado de pobreza extrema. Para dar respuesta a las preguntas de investigación se trazaron los siguientes objetivos analizar los programas Colombia en paz y Colombia equitativa y sin pobreza extrema del Plan Nacional de Desarrollo todos por un nuevo país 2014-2018, a la luz de la teoría del desarrollo de Amartya Sen, frente a la materialización de las apuestas políticas en

Los municipios de San Juan Nepomuceno y el Carmen de Bolívar, Conocer los proyectos y programas que el Plan Nacional de Desarrollo propone y aquellos que se ejecutan en el territorio, para lograr los objetivos propuestos de los programas Colombia en paz y Colombia equitativa y sin pobreza extrema del plan de desarrollo todos por un nuevo país 2014-2018, establecer cuál es la influencia que tiene la teoría de Amatya Sen en los programas Colombia en paz y Colombia equitativa y sin pobreza extrema del plan de desarrollo todos por un nuevo país 2014-2018 en los municipios de San Juan Nepomuceno y el Carmen de Bolívar. 


\section{Metodología.}

La metodología que se utilizará es de tipo explicativo, puesto que requiere adentrarse en terreno para explicar los fenómenos que ocurren en la realidad, así como pretende determinar su origen y causas. Desde lo experimental, es posible plantear la causa-efecto de la problemática.

Es de carácter socio-jurídica, puesto que interpela "los espacios de jurídica, los saberes jurídicos concretos y la práctica jurídica (...) esta puede entonces crear derecho, regular fenómenos culturales que son relevantes para un Estado en un momento histórico determinado". En este sentido está basada en un estudio descriptivo, puesto que su principal objetivo es el análisis de la relación de las propuestas territoriales para la construcción de paz y la erradicación de la pobreza extrema con el PND, y de esta forma diagnosticar el estado de los municipios de San Juan Nepomuceno y el Carmen de Bolívar.

El método que se implementará será el diseño de campo, por medio del cual se analizaran las situaciones que se presenten en los territorios. En este sentido se utilizarán técnicas de recolección de información como la observación, retomada como una estrategia fundamental del enfoque cualitativo, pero a su vez como "un proceso rigurosos de investigación, que permite describir situaciones y/o contrastar hipótesis, siendo por tanto un método científico"(Fernández. 1980, Pág. 135). Esta técnica será orientada a la respuesta concreta de los objetivos específicos. Por tanto, para efectos de esta investigación, será intencionada, selectiva e interpretativa y semi-estructurada, es decir, contará con una guía previamente elaborada que permitirá dar paso o tener acceso a cualquier otra información relacionada con el problema de investigación. 
La entrevista como "la comunicación interpersonal establecida entre el investigador y el sujeto de estudio a fin de obtener respuestas verbales a los interrogantes planteados sobre el problema propuesto. Se considera que este método es más eficaz que el cuestionario, ya que permite obtener una información más completa"(Galán. 2009. Pág.1). Esta también será semi-estructurada, con el ánimo de permitir que los (a) sujetos puedan realizar intervenciones de acuerdo a su perspectiva de la materialización del PND en el territorio.

\section{Resultados y discusiones.}

La primera fase de la investigación se caracteriza por ser un análisis de fuentes secundarias dio como resultados lo que son las primeras bases para el acercamiento a la realidad social. El primero de ellos fueron los planteamientos de Amartya Sen y su teoría de desarrollo que significaron las bases sólidas para la segunda fase de la investigación. El segundo resultado, fue el conocimiento de los planes, programas y proyectos que presentan a nivel nacional y que da pie para el conocimiento de la articulación de estos a un menos nivel como lo es el municipal y por último, la identificación de la teoría del desarrollo de Sen en el plan de desarrollo.

\section{Planteamientos de Amartya Sen y su teoría del desarrollo;}

Generalmente hablar de teorías del desarrollo implica tener el concepto de teorías que se han ocupado de la dinámica de los sistemas industriales en las economías de los países, estas pretenden identificar las condiciones y estructuras socioeconómicas necesarias para hallar una senda de desarrollo humano y crecimiento económico sostenido productivo o no (Cerso, 2006, p1). Ahora bien, el modelo de desarrollo que propone Sen, y que guió esta investigación, aparece como un enfoque alternativo a las concepciones de bienestar que se puedan 
retomar desde otras teorías, referidos a la adquisición de recursos materiales. Sen, denomina su concepción de bienestar desde un enfoque de capacidades básicas, lo que posteriormente nombra desarrollo como libertad, y a partir de aquí amplía su noción, así el desarrollo para este autor puede concebirse: “(...) como un proceso de expansión de las libertades reales de las que disfrutan los individuos" (Graziano, 2002, p.1). Lo que supone, que no es posible medir el desarrollo con otro indicador que no sea el aumento de las libertades de los individuos (Graziano, 2002, p.1), definida por Amartya como "el fin principal del desarrollo", pero también la define como el medio, es decir, “(...) además de ser el objetivo último del desarrollo, la libertad puede ser una excelente herramienta para lograr el mismo. Se trata del papel instrumental de la libertad en el desarrollo"(Graziano, 2002, p.1). Por ello se refiere directamente a los derechos y a la confluencia de los distintos tipos, y argumenta que el aumento de la libertad en el ser humano, fomenta el desarrollo, y que la eficacia de la misma esta en interrelacionar los distintos tipos de libertad. Sen, dentro de su tesis, se apoya en Aristóteles, por lo que entiende la riqueza no como un fin en sí mismo, sino en las cosas que se pueden hacer con ella.

Ahora bien, conceptualizar la pobreza comprende un alto grado de complejidad ya sea por la serie de factores que surgen de esta como la globalización, la educación, la industrialización, la política, etc., o por lo extenso de su significado. En la economía históricamente se ha intentado desde distintos puntos de vista conceptualizar la pobreza, sin embargo economistas como Adam Smith, se centraban más en el problema de la riqueza y dejaban de lado la investigación científica y rigurosa del fenómeno de la pobreza, por lo menos hasta finales de 1940 la economía se dirigía a este enfoque. Después de la segunda guerra mundial y siendo esta desencadenante de nuevas miradas por las distintas problemáticas que surgieron, se empieza a observar en detalle el fenómeno de la pobreza y el subdesarrollo con mayor rigurosidad. Las definiciones que se le otorgan a la pobreza de aquí en adelante, han sido variadas, ser pobre puede considerarse el no disponer de los recursos para obtener los medios mínimos de 
subsistencia o puede ser simplemente hambre (Banco Mundial, 2015. p1). Para Sen, la pobreza no es simplemente la falta de ingreso, sino "(...) la falta de capacidades básicas y la forma de obtenerlo"(Sen, 2000, p.117). Amartya, plantea que existe una subjetividad en cada persona que trata de sobrevivir y que ha experimentado la pobreza, por ello afirma la importancia del desarrollo como libertad a la hora de evaluar al individuo, su sociedad y su papel en ella y defiende la abolición de aspectos de la tradición si esos aspectos conllevan graves pérdidas de libertad y pobreza a las personas que lo sustentan.

La desigualdad entre los componente de la sociedad es lo que genera la pobreza según Sen, esto haciendo referencia específicamente a la mala distribución de las riquezas. No obstante, en el PND en pro de disminuir la desproporcionalidad creó estrategias transversales: competitividad e infraestructura estratégicas; movilidad social; transformación del campo; seguridad, justicia y democracia para la construcción de la paz; buen gobierno y crecimiento verde, puntos fundamentales todos para lograr disminuir la pobreza. Frente a esto, Amartya plantea cinco tipo de libertades que deben ser claves en la superación de la pobreza:

1. Libertades políticas o a todas las capacidades de los colombianos para influir en el manejo de su sociedad.

2. Libertades económicas, o los derechos de una persona a disfrutar de sus recursos económicos y prosperar.

3. Oportunidades sociales o todos los servicios públicos que ofrece una sociedad para la mejora personal de los individuos que la conforman.

4. Garantías de transparencia, que influyen en el grado de confianza que mostrarán los individuos en sus tratos mutuos.

5. Seguridad protectora, para ayudar a los desfavorecidos por cualquier causa a sobrevivir y prosperar en la sociedad. 
Es evidente que no es posible hablar de la erradicación de la pobreza sin la inclusión de los distintos grupos poblacionales (campesinos, afro descendiente, indígenas, etc.) que permitan la participación y las libertades políticas, tampoco sin libertades económicas, sin oportunidades, sin garantías de transparencia y sin seguridad protectora. La historia ha demostrado que la consecución de una paz sostenible está muy relacionado con la eliminación de la pobreza, puesto el conflicto interno en Colombia es una particularidad que no permite conseguir un país sin pobreza por todos los efectos colaterales del mismo y las implicaciones que este genera, por lo que encierra la problemática en un círculo vicioso que no presenta salida alguna.

Sen, nombra distintas faltas de libertad que influyen en el desarrollo de las personas, por ejemplo: el hambre y la desnutrición, la enfermedad por insalubridad y por falta de medios para evitarlas o tratarlas, la falta de libertad política, la denegación de los derechos humanos, la desigualdad entre hombres y mujeres, entre otras (como se citó a Sen, 2004). Así mismo, el autor contempla, que todas las libertades (anteriormente mencionadas) complementan entre sí como medios para conseguir el desarrollo. Es así, como el crecimiento económico además de contemplarse como un medio para incrementar la renta privada, también puede contemplarse como refuerzo para las libertades.

\section{Proyectos y programas que proponen para lograr los objetivos propuestos del programa Colombia equitativa y sin pobreza extrema del plan de desarrollo todos por un nuevo país 2014-2018.}

Determinar cuáles son los principales retos que tiene un nuevo mandatario presidencial puede ser más complicado de lo que parece y más siendo el presidente de un país diverso y con complejos problemas sociales, económicos y políticos, por eso se hace necesario tener una hoja de ruta que permita direccionar la inversión del gobierno nacional partiendo de las necesidades de los habitantes, esta hoja de ruta es el Plan Nacional de Desarrollo. 
Es el documento que sirve de base y provee los lineamientos estratégicos de las políticas públicas formuladas por el Presidente de la República a través de su equipo de Gobierno. Su elaboración, socialización, evaluación y seguimiento es responsabilidad directa del DNP.

EI PND es el instrumento formal y legal por medio del cual se trazan los objetivos del Gobierno permitiendo la subsecuente evaluación de su gestión. De acuerdo con la Constitución política de Colombia de 1991 en su artículo 339 del Título XII: "Del Régimen Económico y de la Hacienda Pública", Capítulo II: "De los planes de desarrollo", el PND se compone por una parte general y un plan de inversiones de las entidades públicas del orden nacional (PND, 2014, P. 18).

El Plan Nacional de Desarrollo 2015- 2018 el cual lleva por nombre "todos por un nuevo país" tiene como propósito generar un país en Equidad en paz y fortalecido en la educación, aspectos que para el gobierno nacional garantizan la sostenibilidad del pueblo colombiano y que llevaría a los niveles de calidad de vida que merece este país. De forma explícita lo determinan apartes del PND La paz, la equidad y la educación conforman un círculo virtuoso. Una sociedad en paz puede focalizar sus esfuerzos en el cierre de brechas y puede invertir recursos en mejorar la cobertura y calidad de su sistema educativo. Una sociedad equitativa en donde todos los habitantes gozan de los mismos derechos y oportunidades permite la convivencia pacífica y facilita las condiciones de formación en capital humano. Finalmente, una sociedad educada cuenta con una fuerza laboral calificada, que recibe los retornos a la educación a través de oportunidades de generación de ingresos y de empleos de calidad, y cuenta con ciudadanos que resuelven sus conflictos sin recurrir a la violencia. De forma más simple: la paz favorece la equidad y la educación, la equidad propicia la paz y la educación, y la educación genera condiciones de paz y equidad. Por ello, este Plan Nacional de Desarrollo se concentra en estos tres pilares, fundamentales para la consolidación 
de los grandes logros de los últimos cuatro años y para el desarrollo de las políticas para los próximos cuatro, en pro de un nuevo país (PND, 2014, p. 29).

Dentro de las principales misiones que tiene el gobierno nacional y el cual está reflejado en el plan nacional de desarrollo es disminuir las brechas de desigualdad y empezar a utilizar las potencialidades de los ciudadanos colombianos de esta manera construir bases solidad que permitan un desarrollo integral hacia el post conflicto, en el capítulo Colombia equitativa y sin pobreza extrema se plantean diferentes objetivos y lineamientos que tienen como columnas vertebrales programas y proyectos para alcanzar estar metas.

\section{Aportes del desarrollo integral de amartya sen en el programa colombia equitativa y sin pobreza extrema del plan de desarrollo todos por un nuevo país 2014-2018.}

Dentro del Plan Nacional de Desarrollo todos por un nuevo país 2014 - 2018 se plantean diferentes estrategias y alternativas que apuntan al desarrollo y que sin duda pueden atender las necesidades de asuntos estructurales como es el caso de la pobreza extrema, el cual tiene un capítulo especial dentro del PND y que especifica en muchos aspectos la continuidad de algunos programa y nuevas estrategias.

Existe una relación de la teoría y del PND que se resaltan, seria arbitrario determinar influencia de la teoría del desarrollo de Sen y del PND desde el análisis documental. Se establece una relación comparativa dentro la visión de Colombia más equitativa y sin pobreza extrema, a través de la promoción el desarrollo humano integral, donde cada uno de los objetivos de desarrollo socioeconómico se articula en la construcción de una sociedad con oportunidades para todos. Sin embargo, no es posible determinar si este desarrollo integral que se propone, basado en oportunidades iguales para todos se materialice en la realidad social. 
Un modelo de desarrollo económico que favorece la equidad debe ser incluyente, aprovechar el talento de todos los colombianos y hacerlos partícipes de los beneficios del crecimiento. Todas las personas, sin importar su procedencia, grupo étnico, género, edad, o discapacidad, deben tener la posibilidad de acceder a mejores servicios públicos, apropiadas condiciones de habitabilidad, vías de acceso para conectarse con las regiones y con el país, un servicio de salud de calidad y una educación que le permita construir su futuro (De Sol citando a Sen, 2004. p.3). Lo que se puede interpretar como ese estado que busca potencializarse y poder brindar una calidad de vida como lo menciona Amartya Sen: existe una diferente forma de interpretar la riqueza y el desarrollo: en vez de verlos por sí mismo únicamente, se les puede contemplar como medios muy poderosos para alcanzar la felicidad, o lo más cercano a esta, como seria vivir mucho y en condiciones óptimas de libertad y bienestar (De Sol citando a Sen, 2004. p.3).

Se hace necesario realizar un bosquejo conceptual y así darle una relación a los aportes de Sen con el PND estos aportes teóricos está en una conexión directa con el objetivo 2 ya expuesto anteriormente así que basados en ese aspecto citaremos los aportes de Sen.

La literatura económica presenta variedad de definiciones del concepto de desarrollo, que abarcan una amplia gama de dimensiones, tanto políticas, sociales, como económicas. En términos generales coinciden en que este concepto hace referencia a factores y cambios cualitativos en la vida del hombre y de la sociedad en la que habita, aunque algunos autores se detienen en el aspecto meramente material del cambio.

En este contexto, Amartya Sen expresó que para hablar del desarrollo de una sociedad hay que analizar la vida de quienes la integran, que no puede considerarse que hay éxito económico sin tener en cuenta la vida de los individuos que conforman la comunidad. El desarrollo es entonces el desarrollo de las 
personas de la sociedad. Por este motivo define concretamente: "El desarrollo es un proceso de expansión de las capacidades de que disfrutan los individuos" (Sen, 2000, p. 123).

Se destaca que la palabra capacidad no es atractiva en sí misma y explica que se ha usado para hablar de las tierras y no de las personas. Sin embargo se elige utilizarla porque le parece clara para expresar las combinaciones alternativas de lo que una persona puede lograr hacer o ser: las capacidades simbolizarían las posibilidades que tienen los individuos de alcanzar desempeños valiosos.

Cabe mencionar que Amartya Sen diferencia el concepto de capacidad humana del tradicional concepto de capital humano. Expresa que si bien ambos conceptos centran su atención en el hombre, la diferencia principal a tener en cuenta está dada porque la literatura económica sobre capital humano pone mayor énfasis en el rol del ser humano como productor de bienes y servicios, por lo que es primordial observar y estudiar cómo cada mejora en la calificación del hombre hace que éste sea más productivo. En cambio, el punto de vista de la capacidad humana planteado por Sen centra su atención en la capacidad de los individuos para vivir la vida, y por ello se analizan los motivos que éstos poseen para valorar y aumentar las alternativas reales entre las cuales poder optar.

Cada persona, en función de sus características, origen y circunstancias socioeconómicas con las que convive, entre otros aspectos, tiene la capacidad para hacer ciertas cosas que valorará por diferentes motivos. Tal valoración puede ser directa o indirecta:

- La primera está en función de aquellos elementos que implican que podrá enriquecer su vida, es decir que le permitirán tener una mejor calidad de vida, como estar bien nutrido o sano.

- La segunda tiene que ver con la posibilidad de contribuir más y mejor en la producción. De esta manera, la perspectiva de la capacidad humana es más 
abarcaría que la teoría del capital humano, esta última es una expresión más limitada de la capacidad humana.

Amartya Sen también se refiere a las capacidades como las libertades fundamentales (o reales) que pueden poseer los individuos. Las define como las diferentes combinaciones de funciones que el individuo puede conseguir y que le permiten lograr distintos estilos de vida. "Por ejemplo, una persona rica que ayune puede conseguir los mismos resultados funcionales en lo que se refiere a comer 0 nutrirse que una persona desfavorecida que se vea obligada a pasar hambre, pero la primera tiene un 'conjunto de capacidades' diferente al de la segunda (la primera puede decidir comer bien y estar bien nutrida, mientras que la segunda no)"

Dentro de los determinantes de las libertades del hombre menciona las instituciones sociales y económicas, como por ejemplo los servicios de educación y salud, o los derechos políticos y humanos, tales como la libertad de expresión y el derecho a elegir las autoridades públicas.

Al mismo tiempo, destaca que las libertades fundamentales integrantes del proceso de desarrollo no sólo son su fin primordial, sino que también forman parte de sus medios principales, están íntimamente relacionadas entre sí y pueden reforzarse unas a otras. "Las libertades políticas (en forma de libertad de expresión y elecciones libres) contribuyen a fomentar la seguridad económica. Las oportunidades sociales (en forma de servicios educativos y sanitarios) facilitan la participación económica. Los servicios económicos (en forma de oportunidades para participar en el comercio y la producción) pueden contribuir a generar riqueza personal y general, así como recursos públicos para financiar servicios sociales" El hecho de que existan libertades y derechos políticos, incluida la libertad de expresión, hace más fácil evitar desastres económicos como las hambrunas.

La educación como capacidad primordial 
Según Amartya Sen hay pruebas suficientes de que, aun con una renta baja, un país que logre que todos sus ciudadanos accedan a la educación y a la asistencia sanitaria, puede obtener muy buenos resultados en cuanto a longevidad y calidad de vida de toda la población. (Sen, 2000, p. 156). No ser capaz de leer, escribir, contar o comunicarse es una tremenda privación y constituye un caso extremo de inseguridad, ya que el individuo tiene la certeza de la privación y la ausencia de cualquier chance de evitar ese destino.

La educación es una capacidad esencial. Cuando la persona es analfabeta su habilidad para entender e invocar sus derechos es limitada, y su falencia educacional puede liderar otra clase de privaciones. Mientras que la educación básica contribuye a reducir la privación básica, la falta de educación tiende a hacer persistir el problema para las personas que están en los estratos sociales más bajos, cuyos derechos no son respetados porque ellos no tienen la habilidad de leer, observar y demandar, ni saben cómo hacerlo. Así, la diferencia educacional se relaciona con la diferencia de clases. Los analfabetos no tienen voz en política para hacer sus demandas.

Al mismo tiempo, la educación permite al individuo socializarse e intercambiar ideas y obtener un empleo, por ello ayuda a aumentar la libertad y disminuir la inseguridad. Amartya Sen relaciona la inseguridad personal con la social, y manifiesta que la misma proviene de muchos caminos, pudiendo la educación tener un rol importante en este aspecto. Las desigualdades en acceso, inclusión y logros escolares conducirán a diferencias en habilidades, que a su vez diferencian la participación de los individuos en el mundo contemporáneo. "Si continuamos dejando a gran parte de la población mundial fuera de la órbita de la educación, vamos a construir un mundo, no solo menos justo, sino también menos seguro" (Sen, 2000, p. 123).

Por otra parte, se destaca que la educación básica tiene un gran rol en la salud. Por ejemplo, con la educación se puede disminuir las epidemias (por medio de la 
disminución de infecciones y por la prevención). Esta afirmación se sustenta en estudios que reflejan que la educación básica posee mayor impacto que la educación especializada en salud.

En este mismo sentido, Sen hace gran hincapié en el rol de la educación de la mujer. Explica que, a medida que la mujer recibe educación y aumenta su capacidad de lectura y escritura, se observa una disminución en las tasas de mortalidad infantil. El autor revela que puede ser mucho más importante el efecto generado sobre la calidad de vida de la población por medio de una mejora en la educación que por medio de la mejora en otras variables que tengan más que ver con el nivel de riqueza de una sociedad. El hecho de que las mujeres reciban educación también contribuye en la disminución de las tasas de fecundidad.

El autor también señala que la educación permite el progreso de un país y menciona ejemplos donde se ha visto que la inversión en educación básica tuvo resultados positivos para el progreso, como Japón, China y Corea del Sur, entre otros. Manifiesta que es difícil participar de la economía mundial exitosamente si la gente no sabe leer y escribir, producir de acuerdo a las especificaciones o tener control de calidad, entre otras consideraciones.

A su vez, señala que por medio de la educación pueden fomentarse actitudes favorables al desarrollo, por ejemplo puede acrecentarse el diálogo social, promoviéndose la estabilidad y la paz dentro de la comunidad, las cuales son deseables por sí mismas, pero además crean un clima favorable para el desarrollo. $Y$ además destaca que la escuela puede influenciar la identidad de la persona, la forma en que la persona se observa a sí misma y a los otros. La naturaleza de la educación es parte de la paz en el mundo. La educación básica tiene un lugar vital en promover la amistad y la lealtad, por ello debe estar disponible para todos, para que los niños reciban ideas de diferentes marcos o puntos de vista, y sean animados a pensar por ellos mismos y con fundamentos. 


\section{Conclusiones.}

Las libertades juegan un papel indispensable en el desarrollo económico de los países, puesto que estas, permiten a su vez el crecimiento económico y lo que podríamos denominar crecimiento social. Para argumentar su postura, cita un dato clave en su obra desarrollo y libertad: “(...) ningún país democrático independiente ha sufrido una hambruna, y es que la libertad política de los ciudadanos se puede traducir en un cambio en el gobierno si el presente no lo hace bien, lo que en última instancia puede obligar a los gobernantes a hacer todo lo posible para garantizar el abastecimiento de alimentos" (De Sol citando a Sen, 2004). Es por lo anterior, que para Sen, aunque todas las libertades son importantes, existe prioridad de una sobre las otras, sobre todo las que nos permiten decidir el futuro de un país.

Para Sen es valioso el crecimiento económico desde los aspectos que tradicionales, dándole prioridad a la industria antes que al ser como tal y a su necesidad de encontrar libertades que lo lleven a ser humanamente más productivo por lo que no es posible hablar de una motivación a la felicidad. Pero con esto no se quiere decir que solo la felicidad llevara al desarrollo económico es fundamental tener en cuenta que un desarrollo sostenible debe ser integral, como también lo expresa Del sol (2004) en su obra Energía, economía y desarrollo sostenible.

El ingreso del (PNB) o de los ingresos individuales, puede desde luego, representar un medio muy importante para ampliar las libertades que disfrutan los miembros de la sociedad, sin embargo, esas libertades dependen también de otros factores, como los planes sociales y económicos (programas para la educación y el cuidado de la salud) y los derechos civiles y políticos (libertad en 
participar en el debate y escrutinios públicos). De la misma forma, la industrialización, el progreso tecnológico o la modernización social pueden contribuir sustancialmente a la expansión de la libertad humana, pero esta depende de otros factores. (Sen, 2009, p. 123).

En sociedades democráticas como la colombiana y más con una fuerte influencia del capitalismo y el neocapitalismo haciendo de esta una sociedad bastante costosa un estilo de vida alto por los aumentos contante en la forma de satisfacer necesidades, Colombia es un país que a pesar de sus riquezas naturales, agrícolas y metalúrgicas sigue siendo un estado con personas en extrema pobreza y con altos índice de desempleo, las estrategias del estado apaciguan de manera general la calidad de vida de los ciudadanos y a pesar de que factores económicos como la inflación estén aumentando y las acciones ya se empiecen a sentir en el caso de los aumentos de la tasa de interés, las medidas son pequeños paños de agua tibia.

Es preciso resaltar que la teoría de Sen en marca en parte lo que se vive en el territorio colombiano y sus principios hacia la libertad es esencial y que la verdadera razón por la cual aún no hemos crecido de la forma como se ha proyectado es por la necesidad de muchos dirigentes de mantenernos atados.

La libertad es esencial para el proceso del desarrollo por dos razones diferentes. 1) La razón evaluativa: La valoración del progreso debe hacerse tomando en cuanta principalmente si mejoran las libertades que tiene la gente. 2) La razón efectividad: La consecución del desarrollo está completamente subordinada al libre albedrío de la gente. Con anterioridad he señalado la primera motivación, la razón evaluativa para enfocarnos en la libertad. Al ir en pos de la otra, la de la efectividad, tenemos que fijarnos en las conexiones empíricas relevantes, en particular las conexiones que refuerzan entre sí las libertades de diversas clases. Es a causa de estas interconexiones que el libre y sustentable albedrío emerge como un importante motor del desarrollo. No sólo es el libre 
albedrío en sí una parte "constitutiva" del desarrollo, sino que también contribuye al fortalecimiento de libre albedrío de otras clases (Sen, 2009, p. 125).

Del desarrollo de esta investigación se concluye en términos generales, las grandes teorías del desarrollo se refieren a condicionantes más relacionados con los ámbitos internacionales o mundiales, o bien nacionales como es el caso objeto de estudio de nuestra investigación sobre desarrollos económicos y sociales para la búsqueda de la igualdad y erradicación de la pobreza extrema. Los componentes centrales del desarrollo humano enfatizan características que ese desarrollo debe tener, es frecuente encontrar en todo nuestro estudio como eje principal el aspecto económico y la relación con conceptos amplios como la pobreza y el desarrollo humano; du enfoque pone el énfasis en el ser humano y en su libertad, libertad como individuo en una sociedad, siendo este su principal medio de desarrollo.

De esta manera, observar el desarrollo desde la óptica del análisis de las libertades substánciales, pone en el centro no solo los medios, sino también los fines del mismo. Se deja para estudios posteriores el análisis detallado de como concebir al desarrollo como un proceso de expansión de las libertades fundamentales.

Si bien la riqueza es la manera de alcanzar una calidad de vida o el medio mediante el cual los individuos la obtienen, también es necesario detectar y tener en cuenta sus limitaciones. El aumento del producto no puede considerarse como único determinante. Éste es tan solo un medio primordial para expandir las libertades de los individuos que viven en sociedad, aunque no el único factor que influye. Cabe resaltar que la riqueza se manifiesta en que permite al individuo conseguir libertades fundamentales.

A la hora de evaluar el bienestar social, Amartya Sen manifiesta y analiza el desarrollo como mejora en la calidad de vida, no solo como viven las personas, 
sino también que alternativas tienen las mismas, se tiene en cuenta el control que las personas tengan de sus vidas. Al hacer referencia del control que tiene los individuos sobre sus vidas, se hace estudia al termino de libertad en el sentido de no privación, ausencias de restricciones particulares e igualdad de oportunidades.

\section{Referencias.}

Banco Mundial. Definiciones de los objetivos de desarrollo del milenio. [En Línea] [http://www.bancomundial.org/temas/omd/definiciones.htm] acceso [05, octubre, 2015]

Bases del plan de desarrollo todos por un nuevo país. Colombia equitativa y $\sin$ pobreza extrema (2015). Encontrado en: https://colaboracion.dnp.gov.co/CDT/Prensa/Bases\%20Plan\%20Nacional\% 20de\%20Desarrollo\%202014-2018.pdf. Acceso: 14 de Noviembre de 2015.

Carvajal Martínez, Jorge Enrique, \& Guzmán Rincón, Andrés Mauricio. (2017). Economía de mercado y democracia: elementos para una crítica al discurso del desarrollo promovido por las Instituciones Financieras Internacionales. Justicia, (31), 116-134. https://dx.doi.org/10.17081/just.22.31.2602

Cerso, Fultado. (2006) Teoría política y desarrollo económico. Encontrado en: http://books.google.es/books?id=7Rpew Lu8C\&printsec=frontcover\&source $=g b s \_g e \_s u m m a r y \_r \& c a d=0 \# v=$ onepage \&q\&f=falseAcceso: 30 de agosto de 2015. México.

Cialti, Pierre-Henri. (2016). La mayor representatividad sindical en España*. Justicia, (30), 17-31. https://dx.doi.org/10.17081/just.21.30.1347 
Del Sol. Jose Gregorio. Energía, economía y desarrollo sostenible. 2004. Capítulo 4. Citando a SEN. Amartya. Desarrollo y libertad.

Fernández Ballesteros. (1980). Métodos de investigación en educación especial. Descargado Link: https://www.uam.es/personal_pdi/stmaria/jmurillo/InvestigacionEE/Presenta ciones/Curso_10/Observacion_trabajo.pdf. Acceso: 26 de mayo de 2016.

Galán, Amador. (2009) Metodología de la investigación. [Artículo de internet]. Link: http://manuelgalan.blogspot.com.co/2009/05/la-entrevista-eninvestigacion.html.

Graziano, Ricardo. (2015) Amartya Sen y el desarrollo como libertad. La viabilidad de una alternativa a las estrategias de promoción del desarrollo. Departamento de Ciencia Política y Gobierno Licenciatura en Estudios Internacionales. 2002. Universidad Torcuato Di tella. Encontrado en: http://www.colombiaaprende.edu.co/html/docentes/1596/articles346050_recurso_3.pdf. Acceso: 30 de agosto de 2015.

Mejía Cáez, Miguel Ramón. (2017). El derecho internacional de los derechos humanos, un nuevo concepto. Justicia, (32), 38-63. https://dx.doi.org/10.17081/just.23.32.2904

Pabón Arrieta, Juan Antonio, \& Torres Argüelles, Alfredo. (2017). La interpretación jurídica en el litigio estratégico en defensa de los derechos humanos en Colombia. Justicia, (32), 227-242. https://dx.doi.org/10.17081/just.22.32.2915

Rodríguez-Burgos, Karla, Martínez Cárdenas, Alán Alejandro, \& Rodríguez-Serpa, Ferney Asdrual. (2017). Estudio empírico sobre los valores democráticos de tolerancia y respeto en la generación milenaria. Justicia, (31), 135-150. https://dx.doi.org/10.17081/just.22.31.2603 
Sanabria, Fabián. (2016). Estado, instituciones democráticas y postconflicto en Colombia*. Justicia, (30), 86-95. https://dx.doi.org/10.17081/just.21.30.1351

Sen, Amartya. (2015) El desarrollo como libertad. México: D - Instituto Nacional de Ecología, 2009. ProQuestebrary. Copyright @ 2009. D - Instituto Nacional de Ecología. All rights reserved. 